\title{
Comparison of heart deformation analysis and cine DENSE in volunteers and heart failure patients
}

\author{
Daniel A Auger ${ }^{1 *}$, Claire Stump ${ }^{1}$, Marie-Pierre Jolly ${ }^{3}$, Andreas Greiser ${ }^{4}$, Kenneth C Bilchick ${ }^{2}$, Frederick H Epstein ${ }^{1,5}$ \\ From 19th Annual SCMR Scientific Sessions \\ Los Angeles, CA, USA. 27-30 January 2016
}

\section{Background}

Post-processing methods have been developed to quantify myocardial strain from steady-state free-precession (SSFP) cine images. In the left ventricle (LV), global strain measurements from SSFP in healthy volunteers ${ }^{1,2}$ and in some types of heart disease compare favorably with myocardial tagging as a reference. We compared heart deformation analysis (HDA) of SSFP images and cine Displacement Encoding with Stimulated Echoes (DENSE) for the assessment of global and segmental circumferential strain (Ecc) and derivative indices in healthy volunteers and heart failure (HF) patients with left bundle branch block (LBBB).

\section{Methods}

Imaging was performed on a 1.5T Siemens Avanto MR system in 3 short-axis planes in 6 volunteers and 29 patients with HF-LBBB. Ecc was computed from SSFP images using HDA (Trufi Strain, Siemens Healthcare,
Erlangen) and from cine DENSE using previously described methods ${ }^{3,4}$. The mean and standard deviation of global Ecc were compared and the correlation between HDA and cine DENSE was assessed. The circumferential uniformity ratio estimate computed with singular value decomposition (CURE-SVD) is a quantitative index of mechanical dyssynchrony based on segmental Ecc, where values near 1 indicate synchrony and lower values indicate dyssynchrony ${ }^{5}$. CURE-SVD was computed for all subjects using HDA and DENSE

\section{Results}

Fig. 1A-B show examples of good agreement of global Ecc between HDA and DENSE for a volunteer and patient, and Fig. 1C shows a good correlation of global Ecc for all subjects. For the segmental analysis, we found greater variability in Ecc (Fig $2 \mathrm{~A}$ ) as assessed by HDA compared to cine DENSE in volunteers $(p=0.001)$, which in some cases mimicked segmental dysfunction,
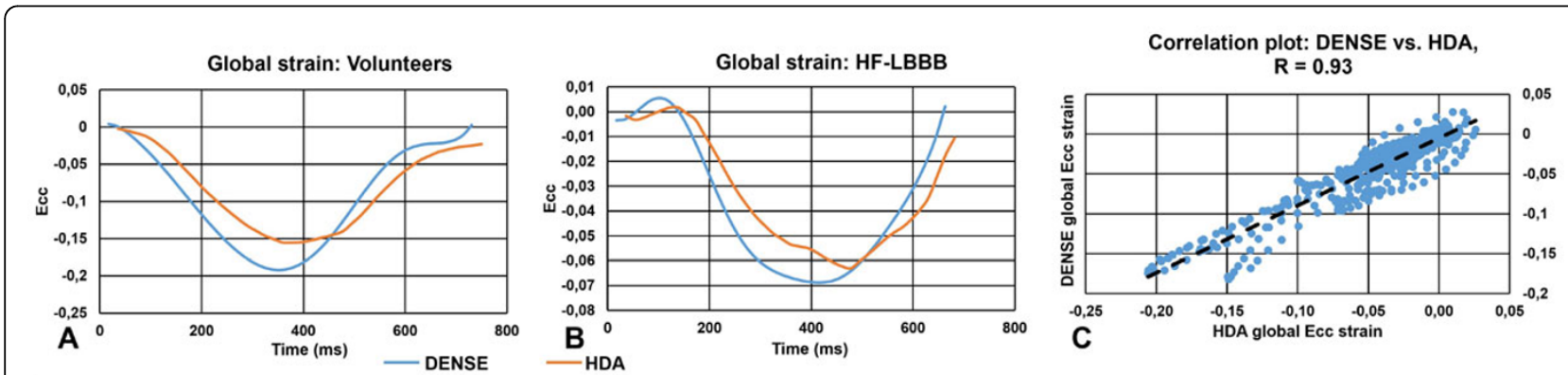

Figure 1 Data shows good agreement between cine DENSE and HDA for global Ecc. (A, B) Cine DENSE and HDA global Ecc curve for healthy volunteer and patient with HF-LBBB. (C) DENSE vs. HDA global strain correlation plot for all data.

'Biomedical Engineering, University of Virginia, Charlottesville, VA, USA

Full list of author information is available at the end of the article 


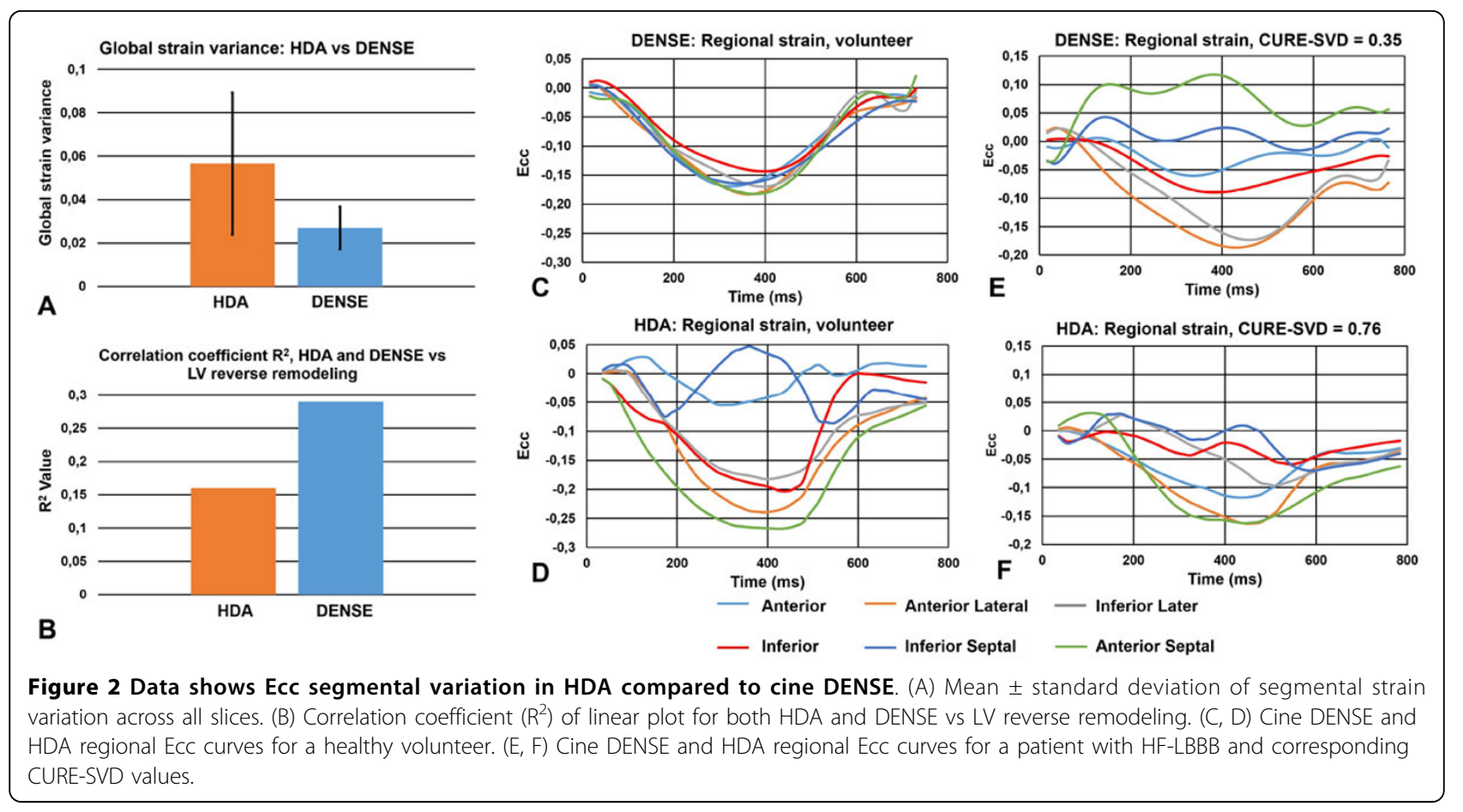

Fig 2B-C. CURE-SVD as assessed using both DENSE and HDA discriminated patients from volunteers. CURE-SVD for volunteers was higher with DENSE than HDA (more synchronous) ( $0.91 \pm 0.0003$ vs $0.82 \pm 0.02, \mathrm{p}<0.05)$ and in patients was lower with DENSE than HDA (more dyssynchronous) $(0.45 \pm 0.03$ vs $0.52 \pm 0.02, \mathrm{p}<0.05)$. An example is shown in Fig 2E-F. Using a linear regression model with CURE-SVD as the predictor and percent change in LV end systolic volume 6 months after CRT as the outcome, DENSE had a better correlation $\left(R^{2}=0.29\right.$, $p=0.002)$ than HDA $\left(R^{2}=0.16, p=0.03\right)$, Fig. 2 A.

\section{Conclusions}

HDA and cine DENSE show good agreement for global Ecc. For regional Ecc, HDA shows greater variability including apparent false-positive segmental dysfunction in some volunteers. While HDA measurements of dyssynchrony can discriminate HF-LBBB from healthy volunteers $(\mathrm{p}<0.01)$, it underestimates the degree of synchrony in volunteers and the degree of dyssynchrony in patients compared to cine DENSE. CURE-SVD by cine DENSE has a better correlation to LV remodeling compared to HDA.

\section{Authors' details}

${ }^{1}$ Biomedical Engineering, University of Virginia, Charlottesville, VA, USA. ${ }^{2}$ Medicine, Cardiovascular Medicine, University of Virginia, Charlottesville, VA, USA. ${ }^{3}$ Medical Imaging Technologies, Siemens Healthcare, Princeton, NJ, USA. ${ }^{4}$ Imaging \& Therapy Magnetic Resonance, Siemens Healthcare, Nürnberg Area, Germany. ${ }^{5}$ Radiology and Medical Imaging, University of Virginia, Charlottesville, VA, USA.
Published: 27 January 2016

\section{References}

1. Hor K et al: JACC 2010, 3(2):P46-51.

2. Morton G, et al: JCMR 2012, 14:43.

3. Spottiswoode, et al: IEEE TMI 2007, 26:15-30.

4. Spottiswoode, et al: MIA 2009, 13:105-115.

5. Ramachandran, et al: Radiology 2015, 275(2):413-20.

doi:10.1186/1532-429X-18-S1-P45

Cite this article as: Auger et al:: Comparison of heart deformation

analysis and cine DENSE in volunteers and heart failure patients. Journal of Cardiovascular Magnetic Resonance 2016 18(Suppl 1):P45.

\section{Submit your next manuscript to BioMed Central} and take full advantage of:

- Convenient online submission

- Thorough peer review

- No space constraints or color figure charges

- Immediate publication on acceptance

- Inclusion in PubMed, CAS, Scopus and Google Scholar

- Research which is freely available for redistribution 